\title{
A measurement study of predicting throughput from LQI and RSSI
}

\author{
Krzysztof Wolosz *, Ulf Bodin, and Laurynas Riliskis \\ Department of Computer Science, \\ Electrical and Space Engineering \\ Luleå University of Technology \\ 97187 Luleå, Sweden \\ \{krzysztof.wolosz, ulf.bodin\}@ltu.se \\ www.ltu.se
}

\begin{abstract}
Wireless sensor networks (WSN) commonly use ZigBee to communicate, especially when low power consumption is demanded. ZigBee may however provide unpredictable throughput although transmission distances are short. This is especially evident in difficult environments with complicated reflections and various materials through which radio signals need to pass through. Distributed scheduling based on cognitive networking principles may improve both network predictability and overall throughput. This paper presents measurements of key parameters for such cognitive scheduling, and discusses their potential for predicting suitable per-node transmission rates. Results include variability of throughput, RSSI and LQI observed for different transmission powers, transmission ranges, and number of transmitting nodes.
\end{abstract}

Keywords: WSN, RSSI, LQI, Throughput, Acknowledgments

\section{Introduction}

Miniaturized and portable wireless sensor nodes are suitable for many different tasks. Such sensors often communicate their sensed data wirelessly and participate for that purpose together with other nodes in wireless sensor networks (WSN). Wireless communication brings many advantages, among them easiness in quickly mounting nodes for various measurement tasks.

This paper focus on communication properties over short distances and evaluates key parameters for predicting transmission capacity between one or more data sources sending data to a common sink. Our results indicate that RSSI can be more reliable than LQI for predicting throughput. RSSI may hence be useful for distributed scheduling based on cognitive networking principles to improve both network predictability and overall throughput.

The paper is organized as follows. Section II describes related work. In section III a survey of the studied transmission parameters is provided. In Section IV

\footnotetext{
* Corresponding author.
} 
we present the tested system and configuration settings used in the experiments, and system validation results. Section V includes measurement results and a discussion of the applicability of these results. Section VI concludes our studies and discuss future work.

\section{Related Work}

Communication properties have been studied in different respects. For example, Jeong and Kim [1] analyze received packets in relation to number of retransmissions and amount of sending nodes. Anastasi et. al. [2], verifies the influence of the weather conditions based on the percentage of received packets. The authors show that the transmission range decreases when fog or rain occurs. Zhou et. al. [3] analyzes packet delivery ratio and throughput considering the novel implementation of Multi-Frequency MAC. The authors state that parallel transmission increases the performance of the WSN. Chehri and Mouftah [4] analyze the throughput from the perspective of number of hops between source and destination. In [5], Dai concentrated on analyzing the throughput according to directional antennas, which allowed to extend the transmission range and increase the network capacity. In [6], the authors verified the correlation between throughput and different implementations of Low Power Listening (LPL).

Papers more directly related to the herein presented work include [7], where Lin et. al. show how to adjust the transmit power during measurements based on RSSI and LQI. The authors found that it is beneficial to control the transmit power of the sending node due to these two parameters. Papers [8] and [9] concentrated on the distance, outdoor and indoor localization impact on the LQI and RSSI indicators. The results showed that it is efficient to utilize two proposed factors in the object tracking methods. In [10], the relationships between RSSI, LQI and Packet Reception Rate were verified.

None of the above-mentioned papers consider the predictability of throughput based on WSN parameters (i.e. RSSI and LQI), which is the focus of this paper.

\section{Parameters Survey}

\subsection{Throughput}

We define throughput as the number of successfully received packets divided by a given period of time in seconds. It is measured in bits per second (bps). The theoretical upper bound throughput for ZigBee is $250 \mathrm{kbps}$, which may be achieved without packets errors and noise. However, transmissions at wireless links are usually interfering each other and are further bothered by noise causing packets loss and followed retransmissions resulting in reduced throughput. 


\subsection{RSSI and LQI}

The two parameters Received Signal Strange Indicator (RSSI) and Link Quality Indicator (LQI) indicate link quality. Both of them are estimated on the physical layer. The particular estimation of these two estimators may however differ between hardware vendors although the intention and purpose of these parameters are the same between vendors and for different type of radios.

RSSI is used to measure the total energy of the received signal defined in $\mathrm{dBm}$. It is obtained when a packet is received and for signals transmitted by the ambient radios. The value of the RSSI is affected by the environment, receiver distance, and variation of transceiver and antenna orientation. RSSI is typically estimated in hardware. The architecture to detect RSSI consists of two main blocks; limiting amplifier and rectifier. The total gain of the limiting amplifier defines the detection range while accuracy is determined by the gain per stage. RSSI is mainly used to measure the distance between nodes and to adjust the receiver gain. The range for the used AT86RF230 radio used in our experiments is from 0 to 28 indexes (-91 dBm to $-10 \mathrm{dBm})$.

LQI defines the reliability of a link from information at packet delivery. It is also known as the Chip Correlation Identificator from how it is estimated. A high LQI corresponds to high Packet Reception Rate. A common usage of the LQI is for controlling the distance of the clusters in WSN. The theory defines several sources for disrupting this parameter; amount of traffic, polarization of electro-magnetic fields or reflections on metallic objects. The estimation of LQI is based on the first 8 symbols of the received physical header. Each symbol represents the sequence of bytes that are compared with the known symbols stored in the embedded table. The correlation of these two values defines the average of the properly received bits. As the result, the reached value is adjusted to Packet Error Rate. For the radio used in our experiments the LQI ranges from 0 to 255 .

\subsection{Transmit power}

In Zigbee AT86RF230 the transmission power can be controlled between +3.0 and $-17.2 \mathrm{dBm}$ which equals $1.99 \mathrm{~mW}$ and $0.02 \mathrm{~mW}$. It influences mainly link quality during the transmission [7]. Sending at maximum power on short distances leads to the interferences between the nodes, which may result in additional packet loss. Also, energy consumption grows with the higher transmission power. Hence, transmission power should be limited and possibly controlled to optimize link throughput and network utilization.

\subsection{Receiver sensitivity}

The receiver sensitivity parameter specifies the minimal possible power of the radio which is able to receive the data. The standard 802.15.4 specifies that the minimum value of this parameter should be greater than $-85 \mathrm{dBm}$ for $2.4 \mathrm{Ghz}$ radios. In the case of the chosen receiver type, it is used the receiver sensitivity level of $-101 \mathrm{dBm}$. 


\subsection{Clear channel assessment mode}

The first mode is called "Energy above threshold". If the measured power level is higher than Energy Detection (ED) threshold than the busy state is indicated. The second mode is related to the characteristics of IEEE 802.15.4 and is described as "Carrier sense only". The detection of the busy state occurs when the radio recognize the modulation and spreading characteristics of the standard. In this case, the ED threshold is not considered. The last mode is called "Carrier sense with energy above threshold". The medium in this case is in the busy state when the power is above ED and the spreading characteristics of the IEEE 802.15.4 are detected.

\subsection{Acknowledgment window size}

One of the methods used to obtain reliable transmission is acknowledging of data and retransmissions in case of loss. Packets which are sent from the transmitter include a sequence number. The receiver sends the acknowledgments in two cases, (1) when it receives the new packet. In this case the payload from the packet is transported to the upper layer. And (2) when the receiver receives the same packet as more than once it sends only the acknowledgment without passing up the payload.

Figure 1(a) shows the procedure for wrongly received acknowledgments by the transceiver. The acknowledgments are missed and the send packets are doubled. The reason is too short acknowledgment window size of waiting time for the acknowledgments. In opposite, Figure 1(b) illustrates the scenario for correctly received acknowledgments.
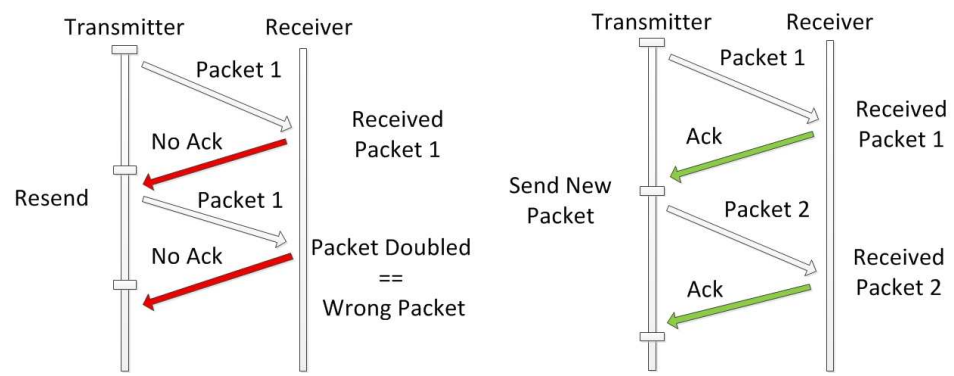

(a) Too short acknowledgment (b) Efficient acknowledgment window size window size

Fig. 1. Acknowledgment procedure for different waiting time for acknowledgments 


\section{System Validation and Test Setup}

Figure 2 illustrates the architecture of the network used for our experiments. The experiments included up to six Mulle sensor nodes serving as transmitters sending data unsynchronized to a single sink node. The base station acts as the broker between wireless nodes and the wired computation platform.

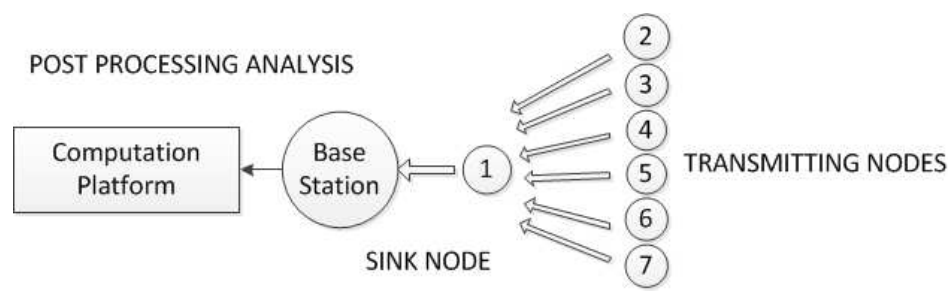

Fig. 2. The architecture built upon 8 nodes used to perform the experiments

The tests were conducted in four steps. Firstly configurations are made, specifying the period of the test (i.e. length in time), receiver sensitivity, transmit power, acknowledgment window size, channel settings and message size. Secondly, the configuration data is sent from the base station to the source nodes that will transmit to the sink node during the experiment. Thirdly, the tests are performed during the defined period. As the experiment is completed, resulting data for the desired statistics is transmitted in sequence from each node to the base station. Finally, the data is accumulated in the computing station which performs the post-process analyze.

The tests were executed during 10 seconds for each configuration. Each test was repeated ten times to get a greater set of samples to analyze. The transmitting nodes were sent unsynchronized as many packets as possible. Packets were sent with PHY and MAC headers of 12 bytes plus varying amount of payload. The payload was varied from 10 bytes and larger. Three nodes were transmitting data expect for experiments with increasing number of transmitting nodes (from 1 to 6 nodes).

All nodes were transmitting channel 11. The clear channel assessment was set to the third mode for carrier sense with energy above threshold. This setting enabled to sense the busy state of the medium. $-101 \mathrm{dBm}$ of receiver sensitivity was determined as the proper energy level for sensing the signal. For the test with fixed transmission power this value was set to the maximum level of +3 $\mathrm{dBm}$.

Figure 3 shows the process of choosing the most appropriate waiting time (threshold) for acknowledgments. As the waiting time for the acknowledgment is lower than 1090 us than the number of acknowledgments received in time drops dramatically, which causes the high number of resent (doubled) packets. We observe that during one second approximately 110 packets is doubled with 
a threshold below 1090 us. However, with a larger window size the number of acknowledgments received in time increases, and the amount of resent packets decreases.

Based on our tests we selected a waiting window size of 1115 us for the experiments. When this value is reached most of the acknowledgments were received in time.

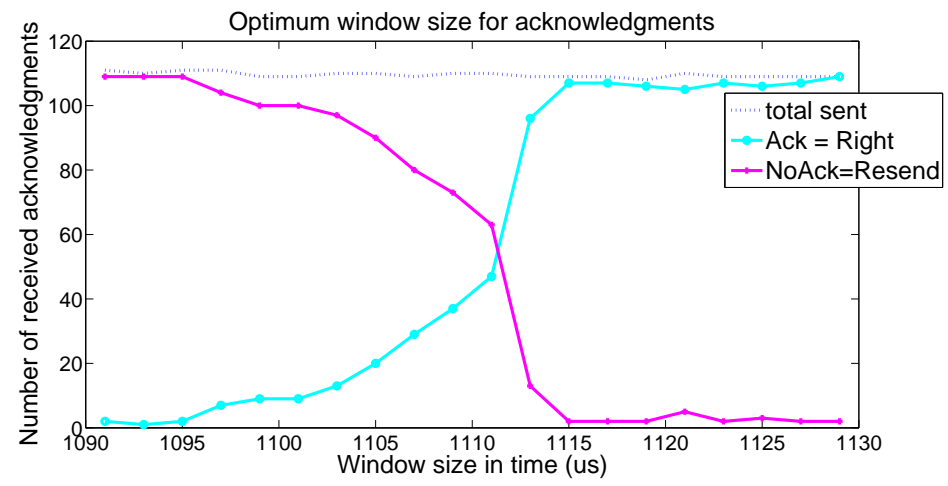

Fig. 3. Number of received acknowledgments for increasing acknowledgment window

The conducted experiments were done indoor. The sensors were placed at the same height. For some tests, the distance between sensors varies in the range of $1 \mathrm{~m}$ to $15 \mathrm{~m}$.

\section{Test Results and Applicability Discussion}

Before looking into relations between throughput and the MAC parameters LQI and RSSI, we study the variability of the throughput. This is to examine at which time scales throughput varies the most and when being more stable. Another property of interest is the distribution to which the varying throughput belongs. Variability and distributions at different time scales are key properties for a cognitive and distributed scheduler we are aiming for as next step based on this paper. These properties are important since we are targeting a scheduler that distributes forwarding capacity at a time scale for which throughput can be statistically predicted from LQI, RSSI or both.

Firstly, we analyze the data due to its distribution. Measurements were performed for throughput, RSSI and LQI. All of the results showed that the data is quite normally distributed. Figure 4 presents the case for the throughput measured for the default settings of $1 \mathrm{~m}$ distance and $+3 \mathrm{dBm}$ of transmit power. The histogram shows that the data is around the central value and the probability plot, which confirms that the data is normally distributed. This result allows us to calculate $95 \%$ confidence intervals for resulting throughput. 

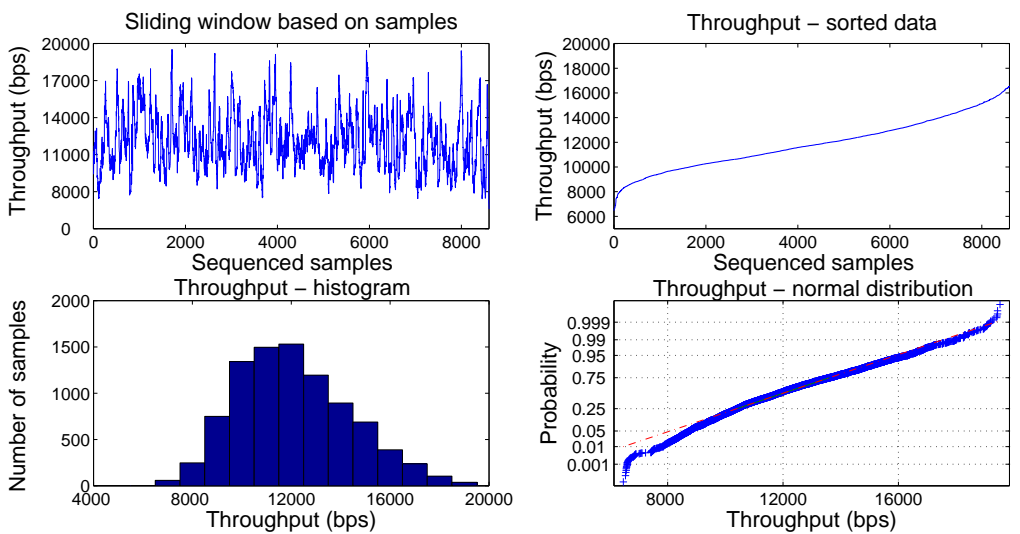

Fig. 4. Analysis of the throughput as the normal distribution

Figure 5 presents the variability of the throughput for different window sizes. Scaling the throughput for 25 samples gives high fluctuation. In the second graph a wider time scale of 400 samples is used. This throughput is considerable smother, i.e. smaller variability. This indicates that we will need to account for variability at shorter times scales when designing the cognitive and distributed scheduler, while throughput at longer time scales may be used as reference, or target values.
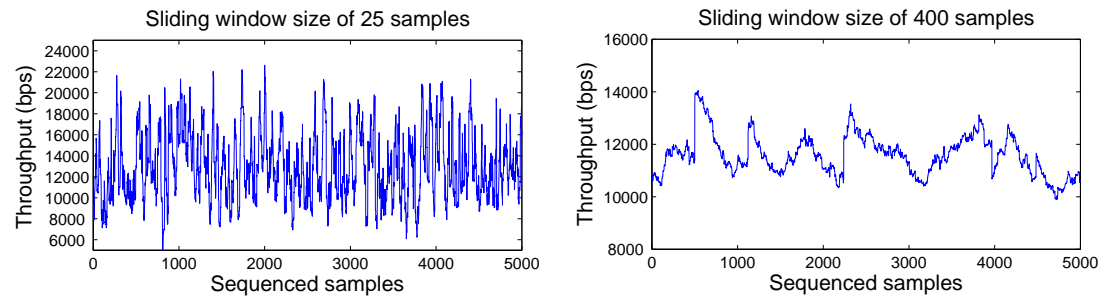

Fig. 5. Throughput variability at different timescales

Figure 6 shows strong dependencies between transmit power, RSSI, LQI and distance. Tests were performed for four increasing distances; $1 \mathrm{~m}, 5 \mathrm{~m}, 10 \mathrm{~m}$ and $15 \mathrm{~m}$. For each distance the transmit power was changed in between $+3 \mathrm{dBm}$ and $-17.2 \mathrm{dBm}$, which corresponds to transmit level index between 0 and 15 . The plotted results depict clear correlation between the measured parameters.

The first graph in Figure 6 shows how the transmission power and distance influence throughput. As expected, the throughput decreases as the distance increase. Our experiments indicate that for the distances of 1 and 5 meters the 
transmit power has lower impact on the throughput than for the distances of 10 and 15 meters. While decreasing the transmit power, the variation of the throughput within 1 meter equals $700 \mathrm{bps}$ and for the distance of 15 meters it is 2050 bps.
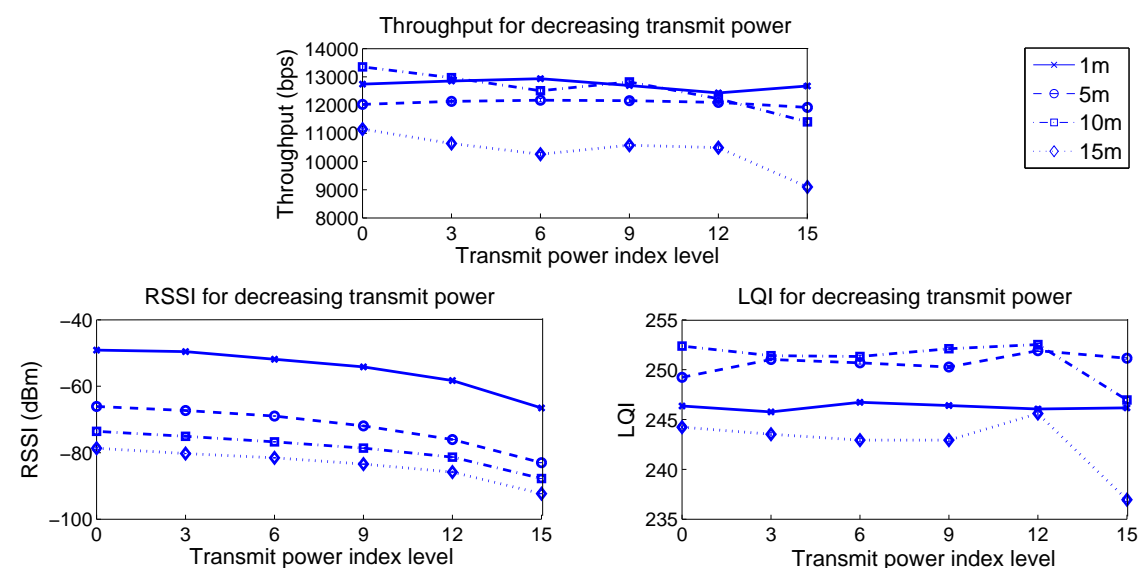

Fig. 6. Transmission Power vs RSSI and LQI for different distances

In the second graph, the RSSI curves decrease with the decreasing transmission power. The curves present the approximately linear tendency. Moreover, the obtained fluctuation range of the RSSI is also lower for the greater distance. RSSI is in the range between -49 to $-66 \mathrm{dBm}$ for $1 \mathrm{~m}$ and -78 to $-92 \mathrm{dBm}$ for $15 \mathrm{~m}$. These results confirm that RSSI is relevant coefficient for estimating the distance between transmitting and sink nodes. The second conclusion is that to reach the highest RSSI is needed the maximum transmission power which leads to high power consumption.

Figure 6 shows the correlation between transmission power and LQI at increasing distance. It can be seen that LQI is relatively stable parameter. It does not change much in between 1 and 5 meters at decreasing transmission power. The variation of this parameter is in the range of 2 indexes. For the greater distance of 10 and 15 meters, the LQI is emphatically getting lower for the decreasing transmission power. For 15 meters and the transmission index level lower than 12, the average LQI equals 244. However, setting the transmission power on the lowest value causes the rapid change to 237. Similar results was obtained for the distance of 15 meters.

Figure 7 shows the fluctuation of RSSI as throughput increases. This result was obtained as follow. Firstly, the throughput was measured. Secondly, for each range of the throughput samples of the RSSI were depicted. We verified that the RSSI is normally distributed and for each range of samples the confidence interval 
was calculated. From this result we can see the exact and strong correlation between the RSSI and throughput.

The observations confirmed that the RSSI increases with the enhancing throughput. According to the confidence interval for each range of the throughput, it is analyzed how many results are overlapping. From this approach the graph is divided on five parts. Each part includes the samples which are of the overlapped intervals. From the perspective of the throughput ranges which aggregate not overlapping intervals indicates that WSN throughput can be predicted from RSSI. Based on this graph we identify RSSI as the most promising parameter to predict throughput because of the non overlapping confidence intervals. With this result we plan to look further into methods of estimating throughput from RSSI.

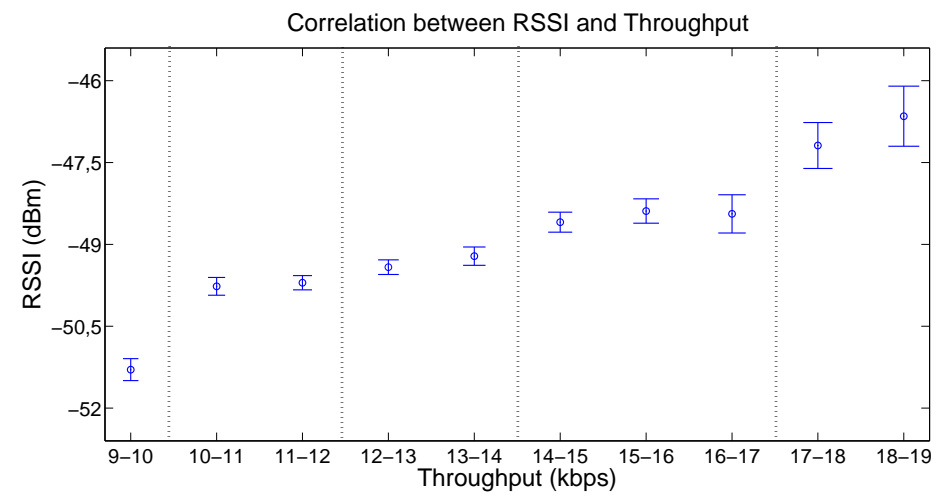

Fig. 7. RSSI presented by confidence interval within increasing ranges of throughput

In Figure 8 we compared the relationship for LQI and throughput using the same method as in the Figure 7. These results indicate that the confidence interval of LQI is much more spread over for each range of the throughput. It leads to often overlapping confidence intervals, which indicates a weaker correlation between throughput and LQI that between throughput and RSSI. This indicates that it is more difficult to predict throughput based on the LQI. There are two reasons for this. Firstly, the background noises during the tests impacting channel quality may cause higher variability in LQI. Secondly, the used hardware it doesn't guaranty the stable functionality.

In the next experiment, the WSN parameters were verified at increasing number of nodes. As shown in Figure 9 the tests are performed for an increasing number of nodes from one to six. At the beginning of the transmission throughput was increasing until two nodes were added in the network. Then, the throughput reached its local maximum. Subsequently, by growing number of the transmitting nodes the throughput was gradually decreasing. For example, for two transmitting nodes the throughput equals $15 \mathrm{kbps}$ and it decreases until $5 \mathrm{kbps}$ for six 


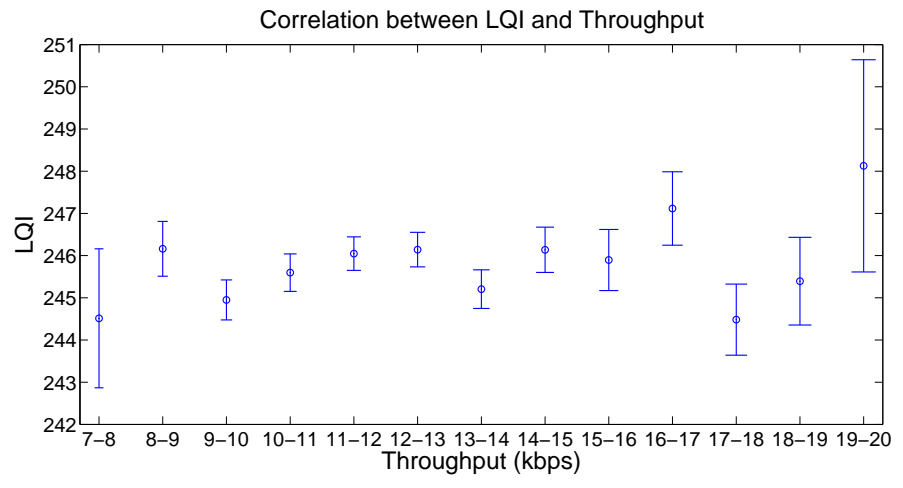

Fig. 8. LQI presented by confidence interval within increasing ranges of throughput

transmitting nodes. The reason for this result is that for the greater number of nodes the sink node is not able to correctly receive that amount of data while transmitted from all nodes. Secondly, the packets are corrupted because of the frequent collisions.

During the tests it was observed a strong correlation between RSSI, LQI and throughput. From 1 to 3 nodes RSSI has the highest value of approximately -54 $\mathrm{dBm}$. Next RSSI drops with the decreasing throughput for three to six nodes. The similar tendency was observed for the LQI. The curve on the graph 9(c) oscillates between 255 and 254 for one and two nodes. The quality of the link is near its the highest performance. However, adding more nodes contributes to significant decrease of LQI. For five and six nodes the LQI is below 245 index level.

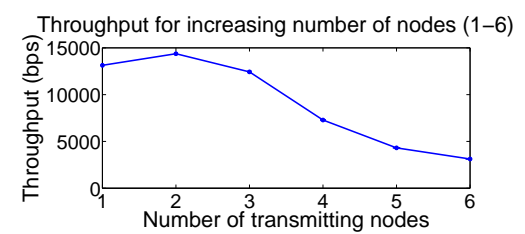

(a) Throughput

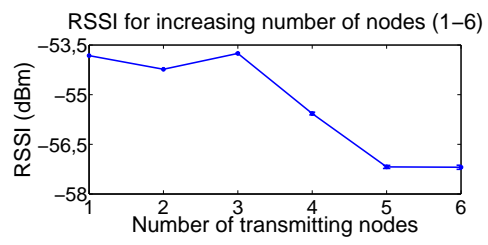

(b) RSSI

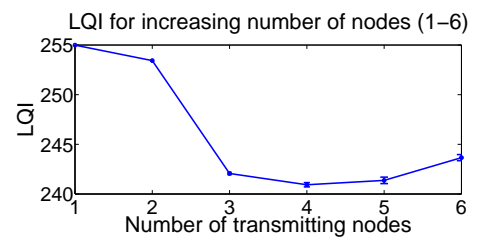

(c) LQI

Fig. 9. Influence of the number of transmitting nodes on the WSN parameters 
In Figure 10 we present the results at increasing amounts of payload. The payload was increased from 10 bytes to 80 bytes. As expected, it can be seen that throughput constantly decreases with increasing payload. For 10 bytes it equals $12 \mathrm{kbps}$ and for 80 bytes the throughput dropped to $4 \mathrm{kbps}$. The reason is that the transmitter and receiver need more time to process larger packets.

According to LQI and RSSI, both parameters provided the similar tendency of slightly increasing until 60-70 bytes and next decreasing for the last two payload sizes. From this we conclude that the increasing sizes of packets cause less corruptions simply because less packets are sent. This leads to lower probability of interference and better quality of the link.

We observed that for packet sizes equal or smaller than 30 bytes the RSSI, LQI and throughput change values rapidly. For the middle sizes of the payload, the characteristics of the graphs are getting much more smother and aligned. Due to these results it is possible to estimate the fluctuations of the throughput based on RSSI and LQI.

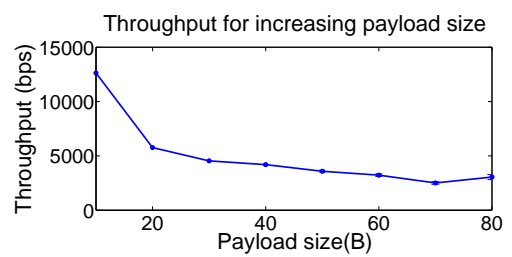

(a) Throughput

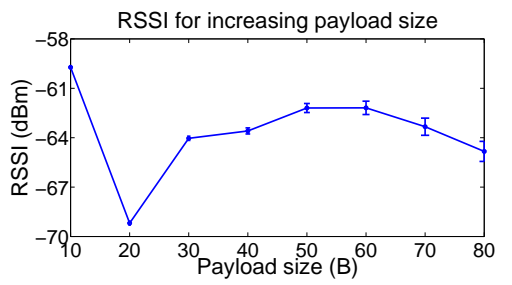

(b) RSSI

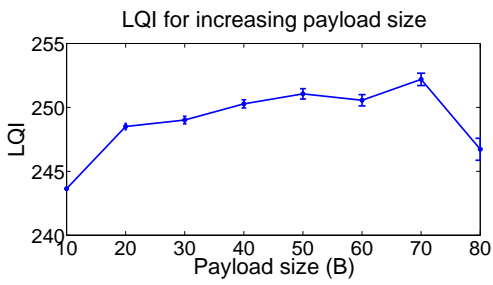

(c) LQI

Fig. 10. Influence of the payload size on the WSN parameters

\section{Conclusions}

In this paper, we have studied correlations between throughput and MAC layer parameter, i.e. LQI and RSSI. We show that throughput is correlated with RSSI and LQI, which indicates that throughput can be predicted from those parameters and may hence prove useful in designing a cognitive and distributed scheduler. Moreover, it was found transmit power, distance, message size and number of nodes impacts the link capacity and resulting throughput of transmissions. 
Based on these results we intend to design a dynamic system that changes configuration based on the analyzed parameters to automatically adapt to its current usage and location of nodes. For such system we believe a cognitive and distributed scheduler to be a key component, making the system stable and predictable for communicating sensed data.

\section{References}

1. Jeong, J. Kim S.: Dot3 Radio Stack. Technical report, CS262A Advanced Topics in Computer Systems Class Project (2002)

2. Anastasi, G., Conti, M., Falchi, A., Gregori, E., Passarella, A.: Performance Measurements of mote Sensor Networks. In: The 7th ACM International Symposium on Modeling, Analysis and Simulation of Wireless and Mobile System, pp. 174-181. ACM New York (2004)

3. Zhou, G., Huang, C., Yan, T., He, T., Stankovic, J. A., Abdelzaher, T. F.: MMSN: Multi-Frequency Media Access Control for Wireless Sensor Networks. In: Proceedings IEEE INFOCOM 2006 25TH IEEE International Conference on Computer Communications, pp. 1-13 (2006)

4. Chehri, A., Mouftah, H.: An empirical link-quality analysis for wireless sensor networks. In: Computing, Networking and Communications (ICNC), 2012 International Conference on, pp. 164 -169. (2012)

5. Dai, H.N.: Throughput and delay in wireless sensor networks using directional antennas. In: Intelligent Sensors, Sensor Networks and Information Processing (ISSNIP), 2009 5th International Conference on, pp. 421-426 (2009)

6. Klues, K., Xing, G., Lu C.: Link layer support for unified radio power management in wireless sensor networks. In: 2007 6th International Symposium on Information Processing in Sensor Networks, pp. 460-469 (2007)

7. Lin, S., Zhang, J., Zhou, G., Gu, L., He T., Stankovic, J. A.: ATPC: adaptive transmission power control for wireless sensor networks. In: Proceedings of the 4th international conference on Embedded networked sensor systems SenSys 06 2007, pp. 223 (2007)

8. Halder, S. J., Park, J. G., Kim, W.: Adaptive Filtering for Indoor Localization using ZIGBEE RSSI and LQI Measurement, Adaptive Filtering Applications. Lino Garcia (Ed.), ISBN: 978-953-307-306-4, InTech (2011)

9. Miluzzo, E., Zheng, X., Fodor K., Campbell, A. T.: Radio characterization of 802.15.4 and its impact on the design of mobile sensor networks. In: Proc. of 5th European Workshop on Wireless Sensor Networks (EWSN), pp. 171-188, Bologna, Italy (2008)

10. Srinivasan, K., Levis, P.: RSSI is Under Appreciated. In: Proceedings of the Third Workshop on Embedded Networked Sensors (EmNets) (2006) 